OPEN ACCESS

Edited by:

He Zhu,

University of Technology Sydney,

Australia

Reviewed by:

Qiong-Sen Wu,

Guangdong University of Technology,

China

Fan Wu,

Southeast University, China

*Correspondence:

Lei Ge

leige@szu.edu.cn

${ }^{\dagger}$ Present Address:

College of Electronics and Information Technology, Shenzhen University,

Shenzhen, China

Specialty section:

This article was submitted to

Radiation Detectors and Imaging,

a section of the journal

Frontiers in Physics

Received: 07 October 2020 Accepted: 20 November 2020

Published: 15 December 2020

Citation:

Gu H, Ge L and Zhang J (2020) A DualBand Dual-Polarized

Omnidirectional Antenna.

Front. Phys. 8:614911.

doi: 10.3389/fphy.2020.614911

\section{A Dual-Band Dual-Polarized Omnidirectional Antenna}

\author{
Hui $\mathrm{Gu}^{\dagger}$, Lei Ge ${ }^{\star \dagger}$ and Jihong Zhang ${ }^{\dagger}$ \\ Shenzhen University, Shenzhen, China
}

A dual-band dual-polarized omnidirectional antenna is investigated. The two bands are generated by a lower circular patch and an upper circular patch, respectively. A set of conductive vias and a coupled ring are utilized to widen the antenna bandwidth. Curved branches are introduced to contribute to circularly polarized radiation. A prototype was designed, fabricated and measured to demonstrate the performance of the antenna. Measured results show that an overlapped bandwidth of $15 \%$ from 2.22 to $2.58 \mathrm{GHz}$ for both $\mathrm{S}_{11} \leq-10 \mathrm{~dB}$ and axial ratio $\leq 3 \mathrm{~dB}$ is obtained in the low band, and an impedance bandwidth of $11.1 \%$ from 5.63 to $6.29 \mathrm{GHz}$ is achieved in the high band. Measured peak gains are approximately $4.3 \mathrm{dBic}$ and $5.4 \mathrm{dBi}$ at two bands. Moreover, omnidirectional radiation patterns are also obtained within the operating band.

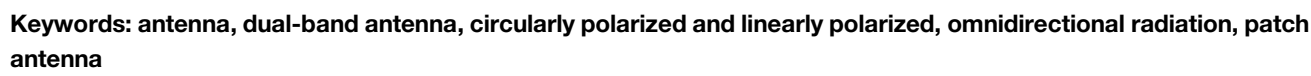
antenna

\section{INTRODUCTION}

Recently, circularly polarized (CP) omnidirectional antennas are of great interest since they can effectively reduce multipath effects, enhance the stability of wireless communication systems and provide a wide signal coverage. Therefore, a large number of efforts have been put into developing $\mathrm{CP}$ omnidirectional antennas [1-8]. In Ref. 1, by introducing shorting vias and vortex slots into a monopolar patch antenna, omnidirectional radiation patterns and wide axial ratio (AR) bandwidth were achieved. Based on dielectric resonator antennas, the designs presented in Refs. 2 and 3 could achieve AR bandwidths of 25.4 and $54.9 \%$, respectively. However, the aforementioned designs all had profiles of more than $0.1 \lambda_{0}$, which are not appropriate for space-limited applications. Patch antennas have advantages of low profile and simple structures, and are therefore suitable for designing $\mathrm{CP}$ omnidirectional antennas [4-8]. For instance, in Refs. 4-6, the antennas loaded with curved branches owned profiles of lower than $0.07 \lambda_{0}$ and AR bandwidths of more than $14 \%$. In Refs. 7 and 8 , by cutting slits on radiating patches and ground planes, $\mathrm{CP}$ omnidirectional radiation performances were obtained and the antenna heights were only 0.028 and $0.026 \lambda_{0}$, respectively.

With the blooming of wireless technologies, antennas with more than one operating band are becoming more and more necessary to satisfy various wireless standards. In Ref. 9, a dual-band CP omnidirectional antenna was proposed. By combining a dielectric resonator antenna and a patch antenna together, the design was able to realize AR bandwidths of 3.16 and 5.06\% at two bands, separately. In Ref. 10 , by utilizing a stacked-patch configuration, a dual-band frequency reconfigurable antenna was presented. In addition, based on artificial $\mu$-negative transmission lines, a dual-band $\mathrm{CP}$ antenna was investigated in Ref. 11. As it is known, CP antennas are required for global positioning systems, satellite communications and linearly polarized antennas are needed for lots of other commercial applications. But few published designs are able to offer dual-band omnidirectional antennas with different polarization states in the two frequency bands. In Ref. 12, a dual-band dual-polarized antenna was proposed. Omnidirectional circular polarization is achieved in the low band and omnidirectional linear 

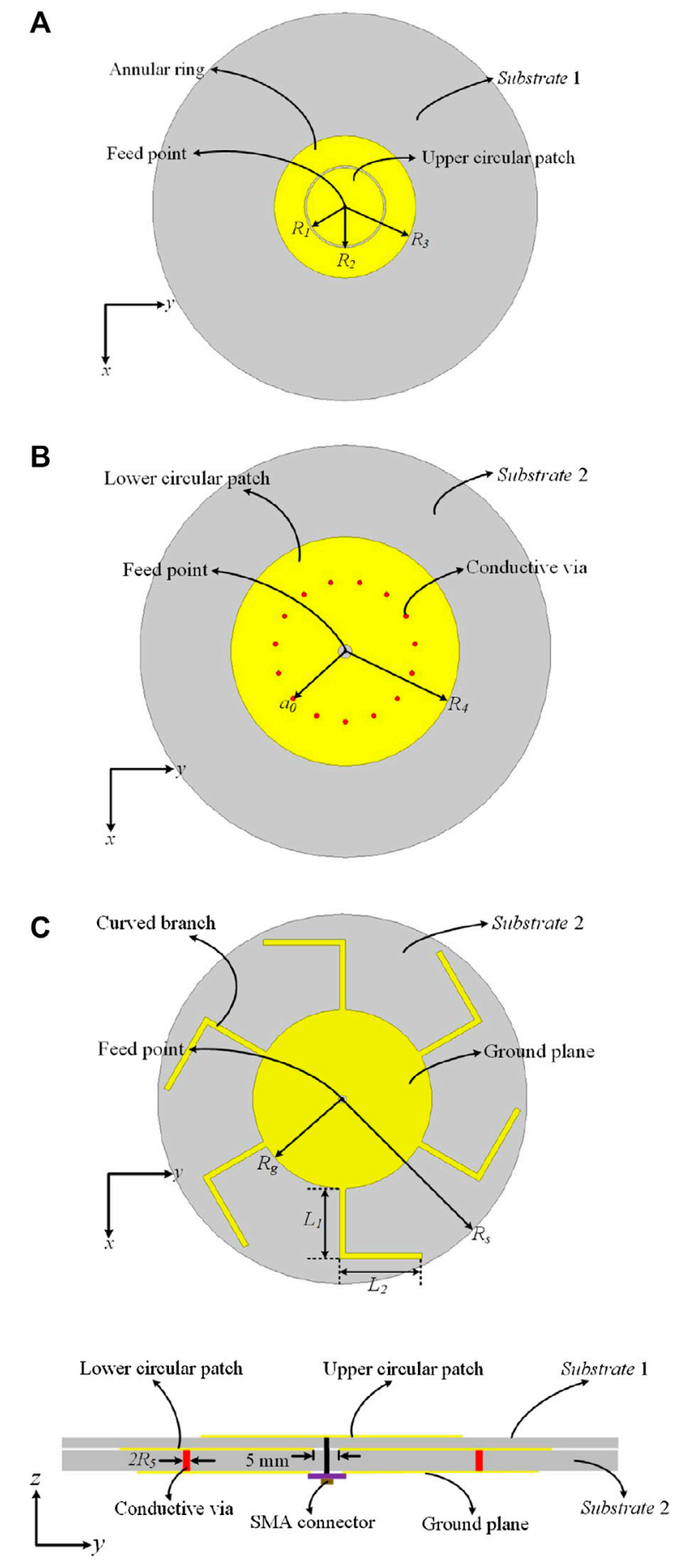

FIGURE 1 | Geometry of the proposed dual-band dual-polarized omnidirectional antenna: (A) Top view of Substrate 1; (B) Top view of Substrate 2; (C) Bottom view of Substrate 2; (D) Side view.

polarization is obtained in the high band. In Ref. 13, a dual-band antenna with different polarization and radiation properties over two bands for vehicular communications is presented. However, the AR bandwidth of the designs $[12,13]$ is too narrow to satisfy the modern wireless systems.
TABLE 1 | Dimensions of the proposed antenna.

\begin{tabular}{lccccc}
\hline Parameters & $\mathbf{R}_{\mathbf{1}}$ & $\mathbf{R}_{\mathbf{2}}$ & $\mathbf{R}_{\mathbf{3}}$ & $\mathbf{R}_{\mathbf{4}}$ & $\mathbf{R}_{\mathbf{5}}$ \\
\hline Values/mm & 17 & 17.3 & 29.3 & 48.3 & 0.7 \\
Parameters & $\mathrm{R}_{\mathrm{g}}$ & $\mathrm{R}_{\mathrm{s}}$ & $\mathrm{L}_{1}$ & $\mathrm{~L}_{2}$ & $\mathrm{a}_{0}$ \\
Values/mm & 43.6 & 90 & 35.2 & 40.1 & 30.7
\end{tabular}

In this paper, a dual-band dual-polarized omnidirectional antenna is presented. The two bands are generated by two circular patches, separately. By adding a set of conductive vias and a coupled ring, the antenna bandwidths are enhanced significantly $[14,15]$. Owing to the introduction of curved branches, a current loop can be formed to provide horizontally polarized field. Together with the vertically polarized field created by the lower circular patch, a CP omnidirectional radiation can be generated in the low band. To verify the design idea, a prototype was designed, fabricated and measured. Both simulated and measured results reveal that wide bandwidths, omnidirectional circular polarization and omnidirectional linear polarization can be achieved.

\section{ANTENNA GEOMETRY AND OPERATING PRINCIPLE}

\section{Antenna Geometry}

Figure 1 shows the geometry of the proposed dual-band dualpolarized omnidirectional antenna and the detailed dimensions are listed in Table 1. As shown in Figure 1, the proposed antenna is mainly composed of an upper circular patch, an annular ring, a lower circular patch, a set of conductive vias, a ground plane, six curved branches and a coaxial probe. The upper circular patch is printed on the top of Substrate 1 (Taconic RF-30, thickness $=1 \mathrm{~mm}, \varepsilon_{r}=3.0$ ) and the annular ring is concentrically placed around the upper circular patch. The lower circular patch and the ground plane are printed on the top and bottom surfaces of Substrate 2 (Rogers 5,870 , thickness $=3.175 \mathrm{~mm}, \varepsilon_{r}=2.33$ ). In order to excite two circular patches simultaneously to achieve a dual-band property, a clearance hole with a radius of $2.5 \mathrm{~mm}$ is etched in the center of the lower circular patch. In this way, the upper circular patch is directly fed by the inner conductor of the coaxial probe and the lower circular patch is fed by the coupling with the clearance hole. As a result, the design is able to operate at two bands. In addition, a set of $N$ conductive vias $(N=15)$ are used to short the lower circular patch to the ground plane and six curved branches are loaded around the ground plane to contribute CP radiation in the low band.

\section{Operating Principle}

As previously stated, by adding the annular ring and a set of conductive vias into the antenna structure, antenna bandwidths can be enhanced dramatically. The curved branches are used to contribute CP radiation. Therefore, in this part, principles of the annular ring, the conductive vias and the curved branches will be further discussed. 

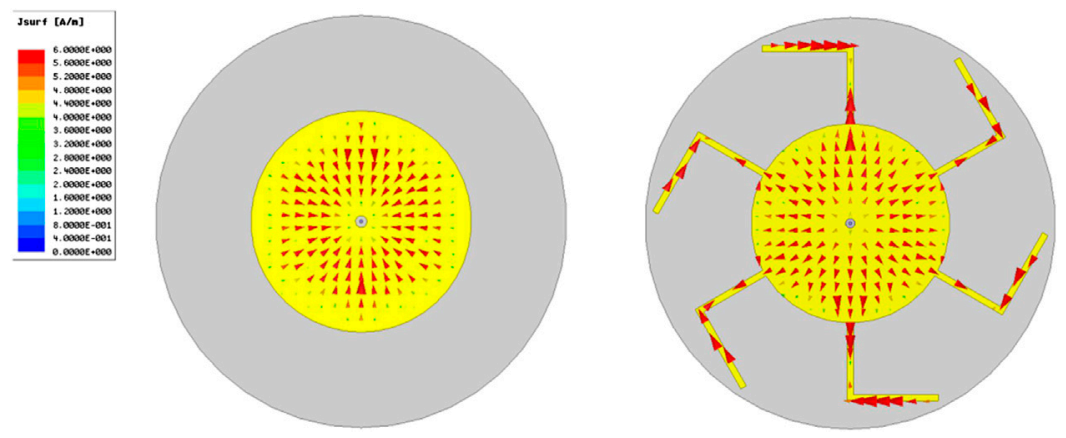

FIGURE 2 | Simulated current distribution on the lower circular patch and ground plane.

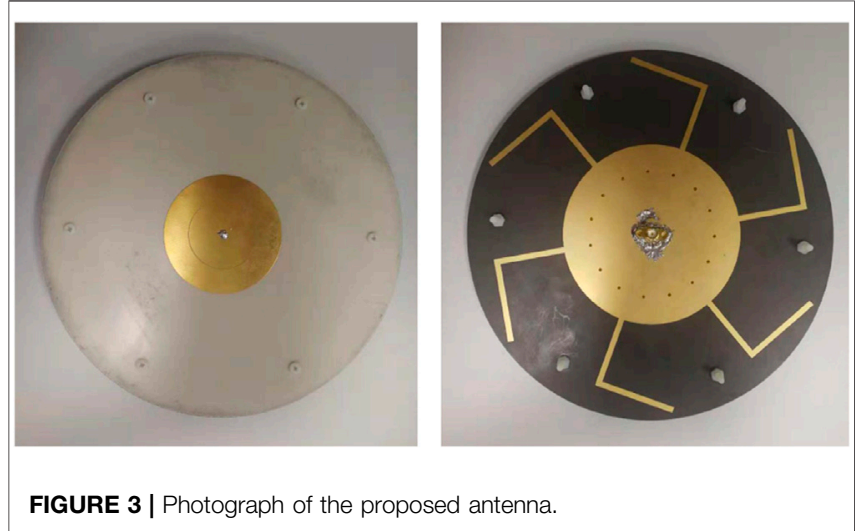

In this design, the annular ring is concentrically placed around the upper circular patch. In consequence, when the upper circular patch is fed, the annular ring can be excited simultaneously by energy coupling. By tuning the radius of the upper circular patch, the size of the annular ring and the distance between them, their resonant frequencies can be moved in proximity to each other. Hence, a wide impedance bandwidth can be obtained in the high band.

As illustrated in Ref. 15, a non-zero resonant frequency of $\mathrm{TM}_{01}$ mode can be created due to the loading of conductive vias. Together with the well-known $\mathrm{TM}_{02}$ mode generated by the lower circular patch, a wide impedance bandwidth can be realized then. The number, radius, and location of the conductive vias have effect on impedance matching of the antenna, and these parameters can be determined based on the design guideline given in Ref. 15.

As it is known, $\mathrm{CP}$ radiation requires two orthogonal electric fields with an equal amplitude and $90^{\circ}$ phase difference. In this design, $\mathrm{TM}_{\mathrm{mn}}$ mode $(\mathrm{m}=0, \mathrm{n}=1,2)$ is generated by the viasloaded lower circular patch, leading to vertically polarized electric field $E_{\theta}$. And the introduction of curved branches can form a current loop around the ground plane, leading to horizontally polarized electric field $E_{\varphi}$. When these two orthogonal fields have an equal amplitude and a quadrature phase difference, CP radiation is achieved in the far field. Figure 2 gives the simulated current distribution on the lower circular patch and the ground plane at $2.4 \mathrm{GHz}$. It is seen that an equivalent vertical current formed by the circular

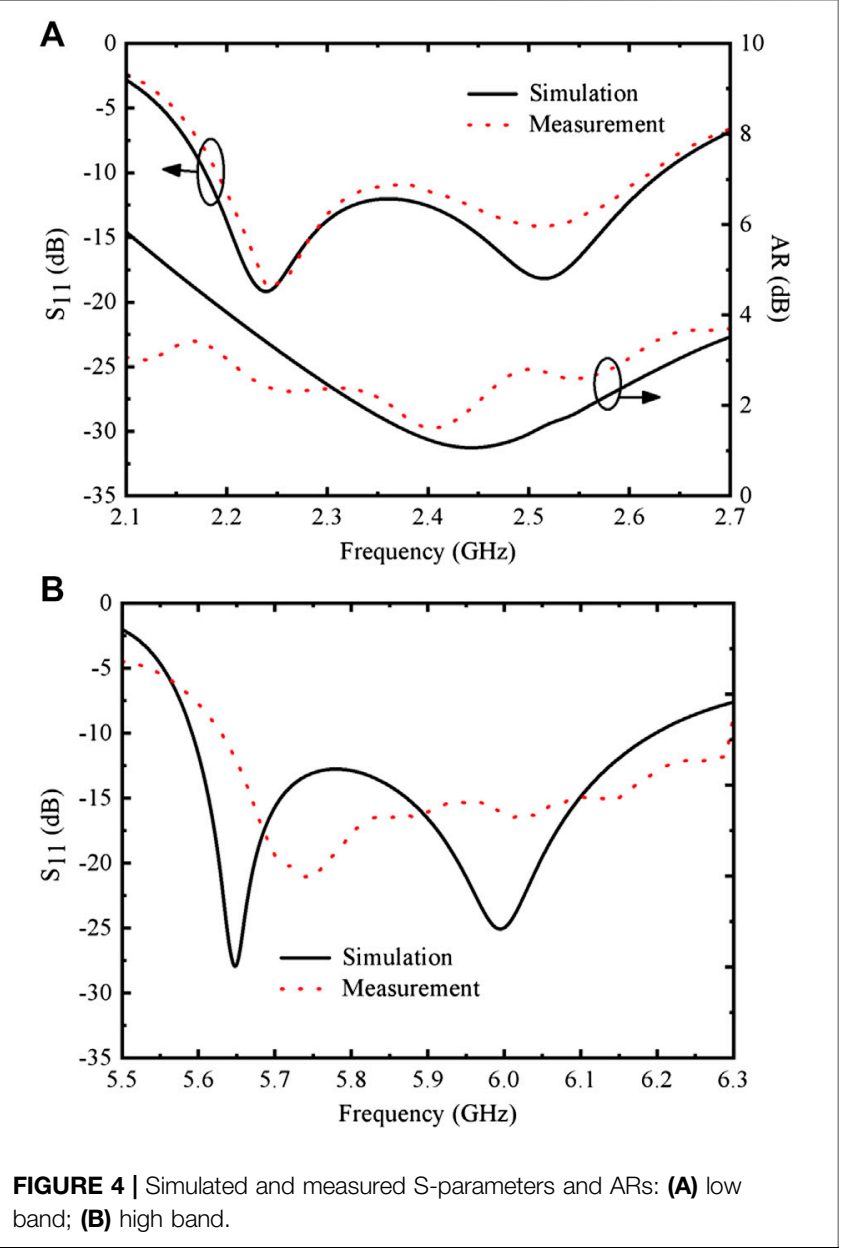

patch and a current loop formed by the curved branch on the ground plane are excited simultaneously. Hence, circular polarization is achieved.

\section{RESULTS}

To verify the proposed design, a fully functional prototype shown in Figure 3 was fabricated and measured. Ansys HFSS, a vector 

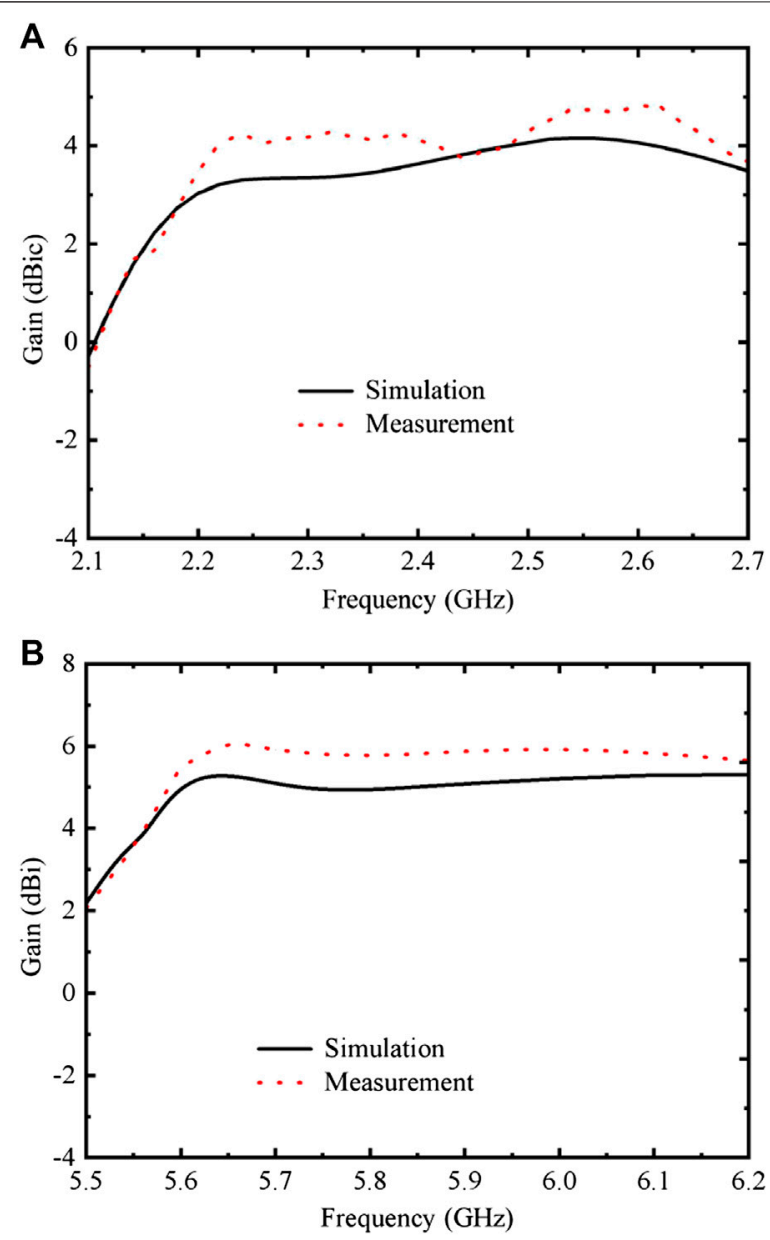

FIGURE 5 | Simulated and measured antenna gains: (A) low band; (B) high band.

network analyzer and a near-field measurement system were used to obtain the simulated and measured results.

Simulated and measured S-parameters and axial ratios (ARs) of the proposed antenna are given in Figure 4. From the figure, it can be seen that the measured impedance bandwidths for $S_{11} \leq$ $-10 \mathrm{~dB}$ are from 2.2 to $2.62 \mathrm{GHz}$ in the low band and from 5.63 to $6.29 \mathrm{GHz}$ in the high band, which agree well with the simulated results. The measured $\mathrm{AR}$ bandwidth for $\mathrm{AR} \leq 3 \mathrm{~dB}$ is from 2.22 to $2.58 \mathrm{GHz}$. Therefore, the effective bandwidths of the proposed design are from 2.22 to $2.58 \mathrm{GHz}$ and $5.63-6.29 \mathrm{GHz}$ at two bands, respectively. Figure 5 shows the simulated and measured peak gains of the proposed antenna. The measured peak gains are approximately $4.3 \mathrm{dBic}$ in the low band and $5.4 \mathrm{dBi}$ in the high band. The discrepancies between the simulated and measured gains are mainly due to the fabrication tolerances and imperfect measurement system.

The simulated and measured radiation patterns in the azimuth plane $\left(\theta=30^{\circ}\right)$ and elevation plane $\left(\varphi=0^{\circ}\right)$ at 2.35, 2.55 and $5.9 \mathrm{GHz}$ are depicted in Figure 6. The measured radiation patterns are in well agreement with simulations. In the low band, omnidirectional radiation patterns can be observed and

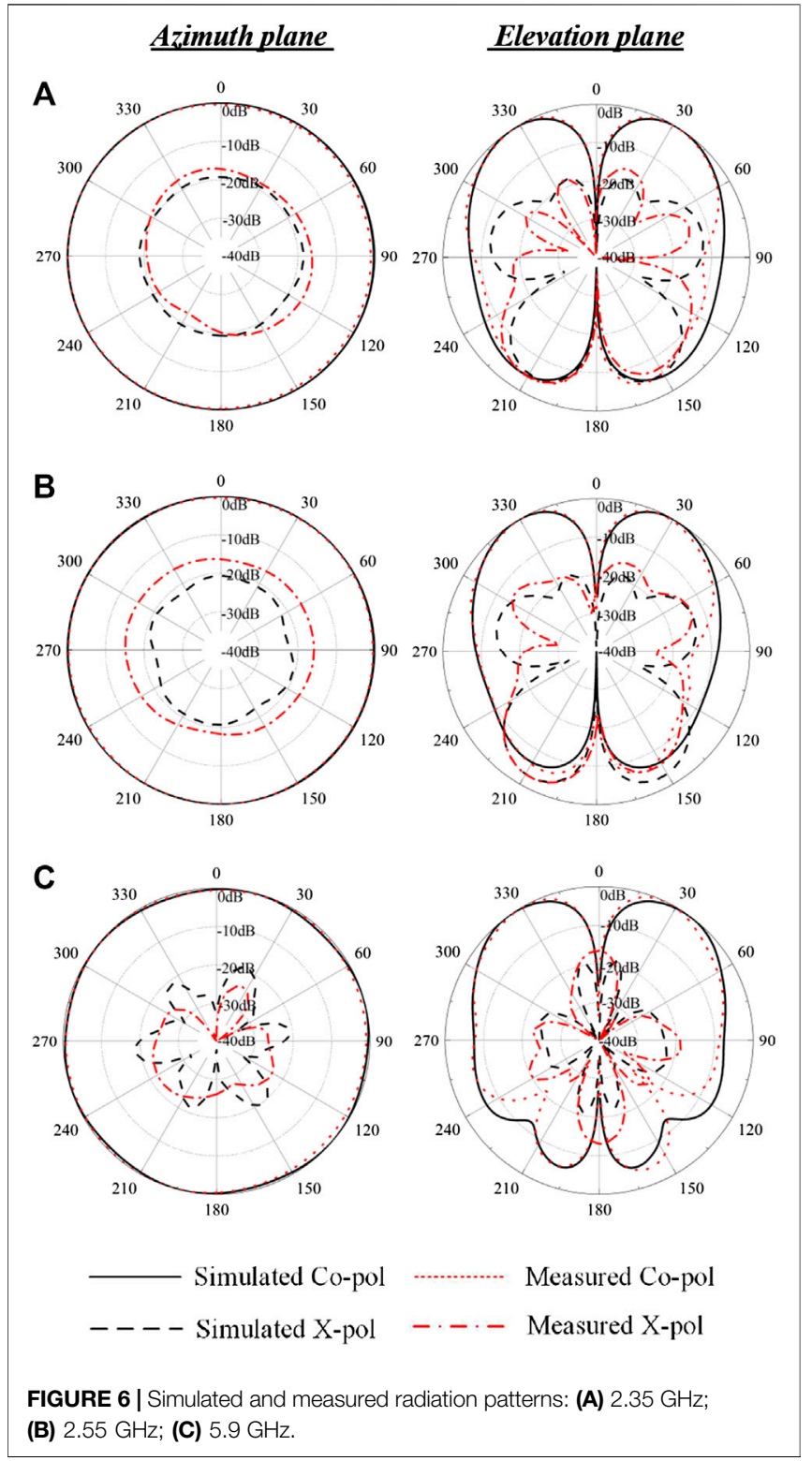

the left-hand (LH) CP fields are almost $15 \mathrm{~dB}$ stronger than the right-hand ( $\mathrm{RH}) \mathrm{CP}$ fields at $\theta=30^{\circ}$ and the whole azimuth plane, demonstrating LHCP radiation is achieved in the far field of the proposed design. In the high band, omnidirectional radiation patterns are also obtained and the cross-polarization levels are lower than $-15 \mathrm{~dB}$ compared with the co-polarization counterpart in both planes. In consequence, a dual-band and dual-polarized antenna with well-controlled omnidirectional radiation patterns is realized in this design.

\section{CONCLUSION}

In this paper, a low-profile dual-band dual-polarized omnidirectional antenna has been proposed. A prototype was constructed and measured to demonstrate the antenna 
performance. The proposed antenna can achieve bandwidths of 15 and $11.1 \%$, gains of approximately $4.3 \mathrm{dBic}$ and $5.4 \mathrm{dBi}$ at two bands, respectively. Besides, omnidirectional radiations with low crosspolarization levels can also be obtained. With these properties, the proposed antenna shows superiorities over the reported designs and is attractive for indoor communication systems.

\section{DATA AVAILABILITY STATEMENT}

The original contributions presented in the study are included in the article/Supplementary Material, further inquiries can be directed to the corresponding author.

\section{REFERENCES}

1. Yu D, Gong SX, Wan YT, Yao YL, Xu YX, Wang FW. Wideband omnidirectional circularly polarized patch antenna based on vortex slots and shorting vias. IEEE Trans Antennas Propag (2014) 62(8):3970-7. doi:10.1109/tap.2014.2325961

2. Pan YM., Leung KW. Wideband circularly polarized dielectric bird-net antenna with conical radiation pattern. IEEE Trans Antennas Propag (2013) 61(2): 563-70. doi:10.1109/TAP.2012.2220101

3. Pan YM, Leung KW. Wideband omnidirectional circularly polarized dielectric resonator antenna with parasitic strips. IEEE Trans Antennas Propag (2012) 60(6):2992-2997. doi:10.1109/TAP.2012.2194678

4. Lin W, Wong H. Circularly polarized conical-beam antenna with wide bandwidth and low profile. IEEE Trans. Antennas Propag. (2014) 62(12): 5974-5982. doi:10.1109/tap.2014.2360223

5. Lin W, Wong H. Polarization reconfigurable wheel-shaped antenna with conical-beam radiation pattern. IEEE Trans Antennas Propag (2015) 63(2): 491-499. doi:10.1109/tap.2014.2381263

6. Chen X, Zhang WM, Han LP, Chen XW, Ma RB, Han GR. Wideband circularly polarized antenna realizing omnidirectional radiation in the wider azimuth planes. IEEE Antennas Wireless Propag Lett (2017) 16(8):2461-2464. doi:10. 1109/lawp.2017.2724082

7. Guo CF, Yang RC, Zhang WM. Compact omnidirectional circularly polarized antenna loaded with complementary V-shaped slits. IEEE Antennas Wireless Propag Lett (2018) 17(9):1593-1597. doi:10.1109/lawp.2018.2856504

8. Shi YZ, Liu JH. Wideband and low-profile omnidirectional circularly polarized antenna with slits and shorting-vias. IEEE Antennas Wireless Propag Lett (2016) 15(3):686-689. doi:10.1109/lawp.2015.2469277

9. Pan YM, Zheng SY, Li WW. Dual-band and dual-sense omnidirectional circularly polarized antenna. IEEE Antennas Wireless Propag Lett (2014) 13(4):706-709. doi:10.1109/lawp.2014.2314744

\section{AUTHOR CONTRIBUTIONS}

All authors listed have made a substantial, direct, and intellectual contribution to the work and approved it for publication.

\section{FUNDING}

This work was supported by the Key Research and Development Project of Guangdong Province (2020B0101080001) and the National Natural Science Foundation of China (62071308). (Corresponding author: LG).

10. Ge L, Li M, Wang J, Gu H. Unidirectional dual-band stacked patch antenna with independent frequency reconfiguration. IEEE Antennas Wireless Propag Lett (2017) 16(2):113-116. doi:10.1109/lawp.2016.2558658

11. Chen X, Han LP, Chen XW, Zhang WM. Dual-Band Circularly Polarized Antenna Using Mu-Negative Transmission Lines. IEEE Antennas Wireless Propag Lett (2018) 17(7):1190-1194. doi:10.1109/lawp.2018. 2838145

12. Liu Y, Li X, Yang L, Liu Y. A dual-polarized dual-band antenna with omnidirectional radiation patterns. IEEE Trans Antennas Propag (2017) 65(8):4259-4262. doi:10.1109/tap.2017.2708093

13. Ge L, Gao S, Li Y, Qin W, Wang J. A low-profile dual-band antenna with different polarization and radiation properties over two bands for vehicular communications. IEEE Trans Veh Technol (2019) 68(1):1004-1008. doi:10. 1109/tvt.2018.2881765

14. Zhang YY, Liu JH, Liang ZX, Long YL. A wide-bandwidth monopolar patch antenna with dual-ring couplers. Int J Antennas Propag. (2014) 13(4):710-713. doi: $10.1155 / 2014 / 980120$

15. Liu JH, Xue Q, Wong H, Lai HW, Long YL. Design and analysis of a low-profile and broadband microstrip monopolar patch antenna. IEEE Trans Antennas Propag (2013) 61(1):11-18. doi:10.1109/tap.2012.2214996

Conflict of Interest: The authors declare that the research was conducted in the absence of any commercial or financial relationships that could be construed as a potential conflict of interest.

Copyright (c) $2020 \mathrm{Gu}$, Ge and Zhang. This is an open-access article distributed under the terms of the Creative Commons Attribution License (CC BY). The use, distribution or reproduction in other forums is permitted, provided the original author(s) and the copyright owner(s) are credited and that the original publication in this journal is cited, in accordance with accepted academic practice. No use, distribution or reproduction is permitted which does not comply with these terms. 